\title{
Overshadowing and blocking between landmark learning and shape learning: the importance of sex differences
}

\author{
Clara A. Rodríguez • V. D. Chamizo • N. J. Mackintosh
}

Published online: 7 April 2011

(C) Psychonomic Society, Inc. 2011

\begin{abstract}
Rats were trained in a triangular-shaped pool to find a hidden platform that maintained a constant relationship with two sources of information, an individual landmark and one part of the pool with a distinctive shape. In Experiment 1, shape learning overshadowed landmark learning but landmark learning did not overshadow shape learning in males, while landmark learning overshadowed shape learning but shape learning did not overshadow landmark learning in females. In Experiment 2, rats were pretrained either with the single landmark relevant or with the shape relevant, in the absence of the alternative cue. Final test trials, without the platform, revealed reciprocal blocking only in females; in males, shape learning blocked landmark learning, but not viceversa (Experiment 2a). In Experiment 2b, male rats received a longer pretraining with the single landmark relevant, and now landmark learning blocked shape learning. The results thus confirm the claim that males and females partially use different types of spatial information when solving spatial tasks. These results also agree with the suggestion that shape learning interacts with landmark learning in much the same way as does learning about any pair of stimuli in a Pavlovian conditioning experiment.
\end{abstract}

\section{A. Rodríguez $(\bowtie) \cdot$ V. D. Chamizo $(\bowtie)$}

Departament de Psicologia Bàsica, Facultat de Psicologia,

Institute for Brain, Cognition and Behavior (IR3C),

Universitat de Barcelona,

Passeig de la Vall d'Hebron 171,

Barcelona 08035, Spain

e-mail: claraarodriguez@ub.edu

e-mail: victoria.diez.chamizo@ub.edu

\section{N. J. Mackintosh}

Department of Experimental Psychology,

University of Cambridge,

Cambridge, UK
Keywords Overshadowing · Blocking · Sex differences . Landmark learning $\cdot$ Shape learning $\cdot$ Morris pool $\cdot$ Rats . Estrous cycle

Experiments on spatial learning have suggested that rats may use a variety of different strategies or cues to find their goal. A common assumption in earlier studies in the Morris pool (Morris, 1981) was that rats use the landmarks outside the pool to find the submerged platform. A subsequent finding reported by Cheng (1986; see also Gallistel, 1990), using a foraging task, implied that rats can use geometric information to locate a hidden goal. He trained male rats in a rectangular arena, where the two short walls of the box and one of the long walls were black, while the other long wall was white. In addition, distinctive visual patterns were placed in each of the box's corners (as well as other nongeometric cues). Food was buried in one corner of the box, and the rats had to search for it. Although rats learned to search in the correct location for the food, they made frequent rotational errors, searching in the corner diagonally across from the one where the food was hidden. The only characteristic that the target corner and the corner diagonal from it shared in common was having one long wall to the left and one short wall to the right, which implies that the information provided by the nongeometric sources of information to find the food location did not seem to be important. Cheng concluded that the rats used the geometric framework of the box itself.

The circular pool used by Morris (1981) does not provide much scope for using geometry to locate the hidden platform. But subsequent experiments by Pearce and his colleagues, employing rectangular, triangular, and kiteshaped pools, have shown that rats will indeed use the shape of the pool to locate the platform (for a review, see 
Pearce, 2009). All these experiments (including the original study by Cheng, 1986) employed male rats only. There is evidence, however, that males and females may differ in the extent to which they rely on geometry or landmarks when navigating (Roof \& Stein, 1999; Williams, Barnett, \& Meck, 1990; a similar sex difference in navigation tasks has been observed in humans-e.g., Chai \& Jacobs, 2009; Saucier, Green, Leason, MacFadden, Bell \& Elias, 2002). Consistent with this, Rodríguez, Torres, Mackintosh, and Chamizo (2010) trained rats in a triangular-shaped pool to find a hidden platform, whose location was defined in terms of two sources of information: one landmark next to the platform, but outside the pool, and one particular corner of the pool. Subsequent test trials without the platform pitted these two sources of information against one another. They revealed that females spent more time in an area of the pool next to the landmark, while males spent more time in the corner of the pool where the platform had originally been located.

If geometrical cues are more salient than landmarks for males but landmarks are more salient than geometry for females, this difference may affect the outcome of experiments in which evidence of cue competition effects between these two sources of information has been looked for. Cheng (1986) initially suggested that geometrical information was processed in a special module that was impenetrable to other sources of information and, therefore, that there should be no competition between geometry and landmarks. Just such a result has recently been reported (e.g., McGregor, Horne, Esber, \& Pearce, 2009), but other experiments have shown evidence of competition between geometry and landmarks (e.g., Horne \& Pearce, 2009; we discuss this literature in more detail in the General discussion section). It seems possible that the outcome of such experiments might be affected by whether male or female rats were employed. In experiments on overshadowing, it is usually the more salient of two cues that overshadows the less salient, rather than vice versa (Mackintosh, 1976). This suggests that landmarks might fail to overshadow geometry in males but would do so in females, while conversely, geometry would successfully overshadow landmarks in males, but not in females. The aim of Experiment 1 was to test this prediction.

\section{Experiment 1}

The term overshadowing refers to the finding that the presence of a second relevant cue will cause animals to learn less about a first than they would have if trained on the first cue in isolation (Pavlov, 1927). In the present experiment, we asked whether this would also be the case when the two cues were a single landmark and a particular corner of a triangular-shaped pool and whether the effect would be the same for males and for females. Two compound groups (with an equal number of males and females in each group) were trained with the platform in one corner of the pool next to a prominent landmark situated immediately outside the pool (see Fig. 1c). Two additional groups (with an equal number of males and females in each group) were trained with a single cue (single-cue groups) - one group, as shown in Fig. 1a, in a circular pool with the platform next to the landmark and the other, as shown in Fig. 1b, in the triangular-shaped pool without the landmark. At the end of training, all the animals

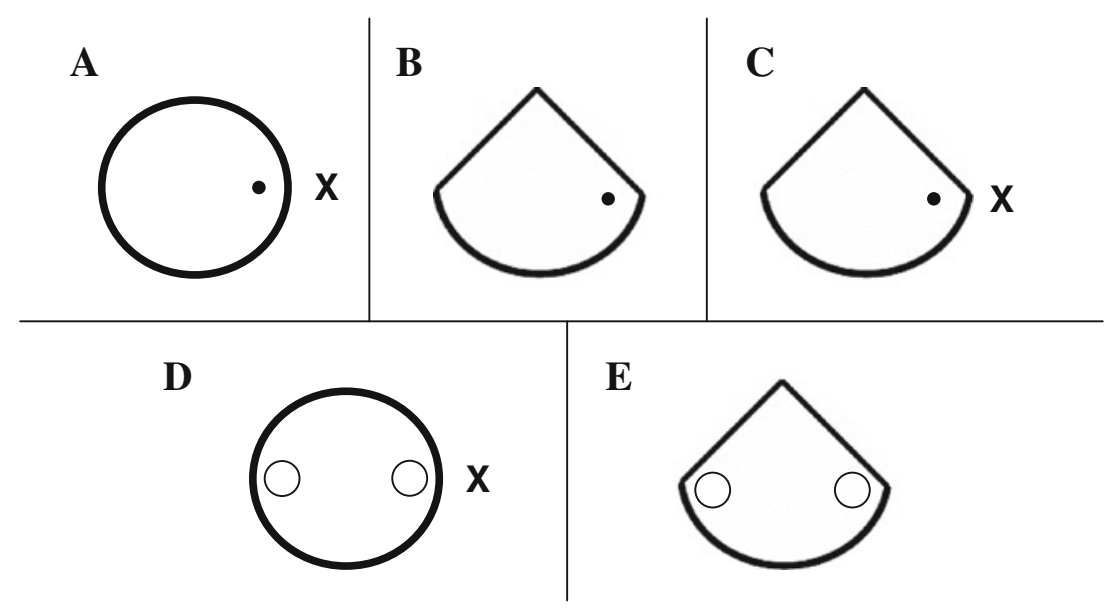

Fig. 1 Top panel: Schematic representation of the pool and the landmark, X, as well as the platform (black circle), during the different types of training trials. The different panels represent the configuration of the pool when training was with the single landmark only (a), with the triangular-shaped pool only (b), and with the compound cue, landmark, and triangular-shaped pool (c) indicating the platform's position. Bottom panel: Schematic representation of the pool and the landmark, X, as well as the two recording areas (target and control), during the two types of test trials. The panels represent the configuration of the pool during the test trial with the single landmark (d) and with the triangular-shaped pool (e) 
received a single test trial without the platform, either in the circular pool with the landmark present or in the triangular pool with no landmark.

\section{Method}

\section{Subjects}

The subjects were 64 naive Long Evans rats, 32 males and 32 females, approximately 4 months old at the beginning of the experiment. The rats were caged ingroups of 2 , and they were maintained on ad lib food and water, in a colony room that had a 12:12-h light:dark cycle. They were tested within the first $8 \mathrm{~h}$ of the light cycle.

\section{Apparatus}

The apparatus was a circular swimming pool, made of plastic and fibre glass, as used by Morris (1981). It measured $1.58 \mathrm{~m}$ in diameter and $0.65 \mathrm{~m}$ deep and was filled to a depth of $0.49 \mathrm{~m}$ with water rendered opaque by the addition of $1 \mathrm{cl} / 1$ of latex. The water temperature was maintained at $22^{\circ} \pm 1{ }^{\circ} \mathrm{C}$. The pool was situated in the middle of a large room, mounted on a wooden platform $0.43 \mathrm{~m}$ above the floor. The pool could be used as a circular pool or as a triangular-shaped pool. To create the unconventional pool shape, two acrylic boards forming an angle of $90^{\circ}$ were inserted in the pool, with platforms at the base that supported them vertically. The boards were $39.5 \mathrm{~cm}$ high, $0.5 \mathrm{~cm}$ thick, and $112 \mathrm{~cm}$ long. The height of the top of the panels coincided with the pool border. The pool was surrounded by black curtains reaching from ceiling to the base of the pool, forming a circular enclosure $2.4 \mathrm{~m}$ in diameter. Inside the black enclosure, above the border of the pool, a single object could be placed. It was suspended from a false ceiling, $35 \mathrm{~cm}$ above the surface of the water. For all the rats, the object, landmark X, was a skittle $(6 \mathrm{~cm}$ in diameter at the base and $16.5 \mathrm{~cm}$ in height, with the wider part measuring $26 \mathrm{~cm}$ in circumference, with blue and yellow segments). This single landmark (Fig. 1a) or the point formed by a straight wall to the left and a circular wall to the right of the two added boards (Fig. 1b), as well as the combination of these two sources of information (Fig. 1c), could define the location of the platform. In order to ensure that the rats used the landmark and shape of the pool, rather than any inadvertently remaining static room cues (such as noises from pipes and air conditioning), to locate the platform, the landmark, the two boards, and the platform were semirandomly rotated with respect to the room $\left(90^{\circ}, 180^{\circ}, 270^{\circ}\right.$, or $\left.360^{\circ}\right)$, with the restriction that all four positions were used each day. A closed-circuit video camera with a wide-angle lens was mounted $1.75 \mathrm{~m}$ above the center of the pool inside the false ceiling, and its picture was relayed to recording equipment in an adjacent room. A circular platform, $0.11 \mathrm{~m}$ in diameter and made of transparent Perspex, was mounted on a rod and base and could be placed $0.38 \mathrm{~m}$ from the point and landmark on a line that bisected the center of the pool, with its top $1 \mathrm{~cm}$ below the surface of the water. Throughout phase 1 and phase 2, the two cues (landmark and shape) were rotated between trials, and the platform always rotated with them.

\section{Procedure}

There were four types of trials: pretraining, single-cue training, compound-cue training, and tests. Pretraining consisted of placing a rat into the circular pool without the landmark or boards, but with the hidden platform present. The rat was given $120 \mathrm{~s}$ to find the platform, and once the rat had found it, it was allowed to stay on it for $30 \mathrm{~s}$. If it had not found the platform within the $120 \mathrm{~s}$, it was picked up, placed on the platform, and left there for $30 \mathrm{~s}$. Rats were given five such pretraining trials over 2 days, with two trials on day 1 and three on day 2. The platform was moved from one trial to the next, and the rat was placed in the pool in a different location on each trial, as far as possible equally often on the same side of the pool as the platform or on the opposite side, and with the platform to the right or to the left of where the rat was placed. The rats were run in groups of 8 and spent the $8-10 \mathrm{~min}$ of the intertrial-interval (ITI) in small individual compartments.

For training, rats were divided into four groups of 16 animals each (i.e., 8 males and 8 females per group): compound-L, compound-S, and two single-cue groups (landmark and shape). The compound-L and compound-S groups received 40 trials of compound-cue training for 5 days, 8 trials daily (i.e., learning was with the single landmark in the triangular-shaped pool, both predicting the platform's position, as shown in Fig. 1c), while the landmark and shape groups received 40 trials of singlecue training for 5 days, 8 trials daily (i.e., the landmark group was trained with the landmark X in the circular pool, while the shape group was trained in the triangular-shaped pool, in the absence of the landmark, as shown in Figs. 1a and $1 \mathrm{~b}$, respectively). At the end of the training phase, all the groups received a test day. This test day consisted of 8 training trials, which were identical to the training trials for each group, followed by 1 test trial, without the platform, which was $30 \mathrm{~s}$ long. Rats in the compound-S and shape groups were tested without the landmark in the triangular-shaped pool, while rats in the compound-L and landmark groups were tested with the single landmark in the circular pool. For purposes of recording the rats' behavior, two areas of the pool were chosen $(0.22 \mathrm{~m}$ in diameter; twice the hidden platform diameter), one in front 
of the target cue (i.e., either the landmark in the circular pool or the correct corner of the triangular-shaped pool), and the other one, for control reasons, $180^{\circ}$ apart (as shown in Figs. 1d and 1e).

In addition, we also examined the possibility that the estrous cycle of female rats could influence their performance (see the Appendix for details).

Results and discussion

Figure 2 shows the latencies for finding the platform by males and females in all groups throughout cue learning. An ANOVA conducted on the training trials, with group (compound-S, compound-L, shape, and landmark), malefemale, and days (1-5) as factors, revealed that the days, $F$ $(4,224)=119.90$, and group, $F(3,56)=3.92$, variables were significant, as well as the days $\times$ group and days $\times$ male-female interactions, $F_{\mathrm{s}}(4,224)=1.89$ and 8.13, respectively. Simple main effects of the days $\times$ groupinteraction showed that the groups differed on day 1 only, $F$ $(3,60)=4.42$. Subsequent comparisons between groups (Newman-Keuls) revealed that the shape group differed from the other groups, compound-S, compound-L, and landmark, which did not differ between them. Simple main effects of the days $\times$ male-female interaction revealed that the male-female variable differed on day 1 only, $F(1,62)=$ 9.16, reflecting the fact that males reached the platform faster than did females. No other main effect or interaction was significant, $F_{\mathrm{S}}<2.0$. All the rats improved their performance as training progressed. An ANOVA of the escape trials during the test day (day 6), with group (compound-S, compound-L, shape, and landmark) and male-female as factors, revealed that no main effects or interactions were significant, $\left(F_{\mathrm{s}}<3.5\right)$.

Figure 3 shows the time spent in the two recording areas (i.e., target and control) by the four main groups (data for the males and females within each group being shown separately) during the 30 -s test trials (i.e., shape test and landmark test [triangular and circular pools, respectively]). Preliminary Student $t$-tests were used to compare the performance of each group in the two areas with chance in order to evaluate whether the test results reflected significant spatial learning. The chance level was obtained by calculating the spacepercentage of the recording areas (i.e., target and control areas) in relation to the total area of the pools (i.e., circular pool and triangular-shaped pool). From those percentages, the proportion of time that an animal would have spent in both the target and control areas in each pool while swimming around was calculated. In the circular pool, the level of chance was $0.58 \mathrm{~s}$; in the triangular-shaped pool, $0.88 \mathrm{~s}$. Males and females in all the groups differed from chance in the target areas, $t \mathrm{~s}(7)=16.04,16.25,14.55,19.81,200.74$, $17.66,29.78$, and 20.45 , for compound-S males, compound-S females, shape males, shape females, compound-L males, compound-L females, landmark males, and landmark females, respectively, while none of them differed from chance in the control areas, $(t \mathrm{~s}<2.5)$. Consequently, no further analyses were performed with the results from the control areas. An ANOVA on the time spent in the target areas with group (compound, single), male-female, and type of cue tested (landmark or shape) as factors revealed that the group and type of cue tested variables were significant, $F_{\mathrm{s}}(1$, $56)=37.60$ and 15.36 , respectively; the group $\times$ cue and cue $\times$ male-female interactions were also significant, $F_{\mathrm{s}}(1$, $56)=8.91$ and 52.50, respectively, as well as the triple group $\times$ cue $\times$ male-female interaction, $F(1,56)=21.42$. The analysis of the triple male-female $\times$ cue $\times$ group interaction showed that on both tests (shape and landmark), the group $\times$ male-female interaction was significant, $F_{\mathrm{s}}(1,28)=4.85$ and 18.19 , respectively. Simple main effects of these interactions revealed that on the shape test trial, the group variable was significant in females only, $F(1,14)=104.15$, reflecting that the group trained with the single cue (shape) spent more time searching in the target area than did the group trained with the compound cue (compound-S); the two groups did not differ in males, $(F<1.5)$. On the landmark test trial, the group
Fig. 2 Mean escape latencies by the four groups (i.e., males and females separately) in Experiment 1 during the training phase and the test day. Errors bars denote standard errors of the means

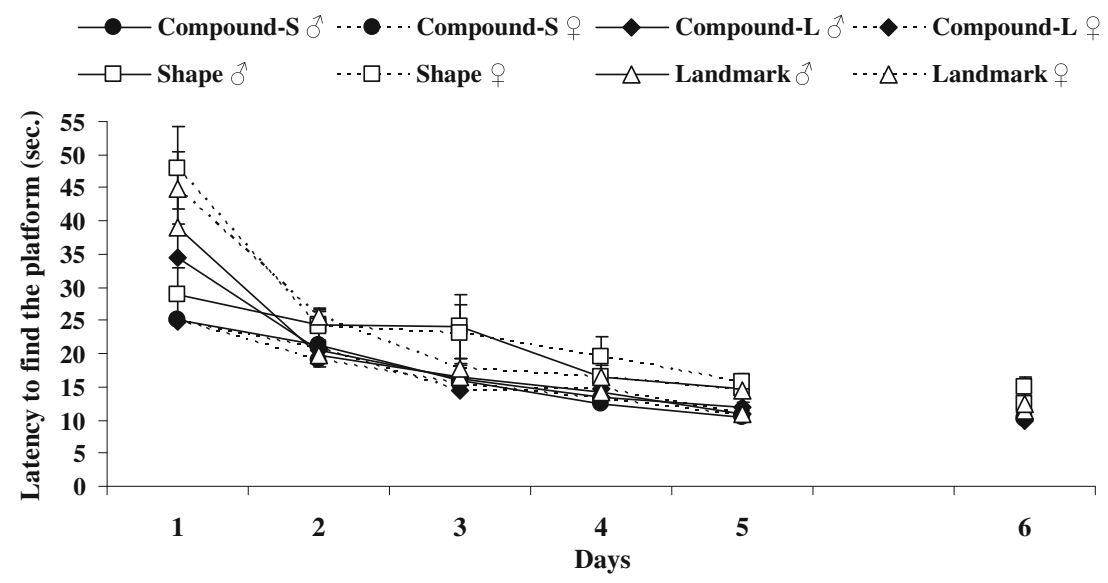


Fig. 3 Mean time spent in the two recording areas (i.e., target and control) by the four separately) during the test trials (i.e., shape test and landmark test[triangular and circular pools, respectively]) in Experiment 1. Errors bars denote standard errors of the means groups (i.e., males and females

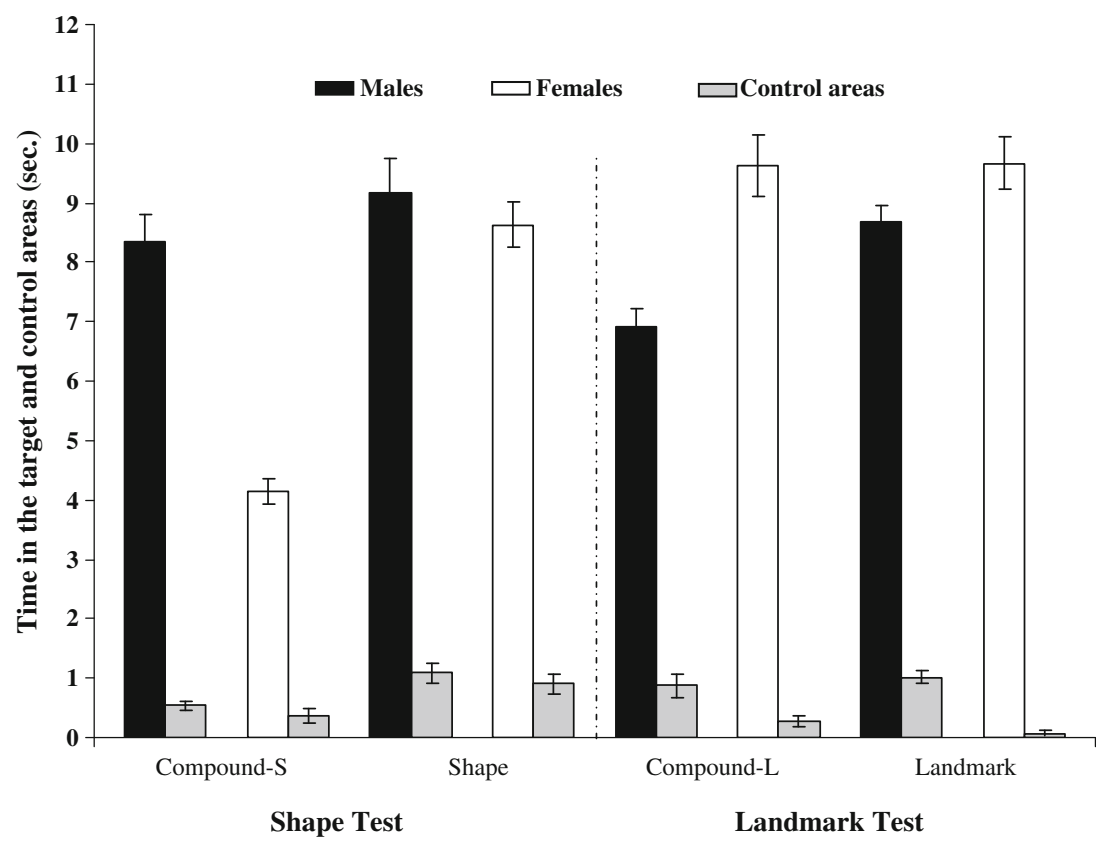

variable was significant in males only, $F(1,14)=19.18$, reflecting that the group trained with the single cue (landmark) spent more time searching in the target area than did the group trained with the compound cue (compound-L); the two groups did not differ in females $(F<0.5)$. These results indicate that landmark learning overshadowed shape learning in females, but not in males, while shape learning overshadowed landmark learning in males, but not in females.

In summary, an overshadowing effect was found between geometry and landmark learning. Evidence of overshadowing was provided by the superior performance of single groups, by comparison with compound groups. More important, overshadowing was asymmetrical, both in males and in females. Specifically, in males, shape learning overshadowed landmark learning, but not viceversa, while in females, landmark learning overshadowed shape learning, but not viceversa.

\section{Experiment 2a}

A second example of cue competition effects is blocking, which is often observed when prior establishment of one element of a compound cue as a signal for reinforcement reduces or blocks the amount learned about the other cue (Kamin, 1969). In Kamin's original experiments, and many since, blocking has been reciprocal, in the sense that whichever of the two cues is pretrained as a signal for reinforcement is able to block learning about the other. But there are exceptions: Hall, Mackintosh, Goodall, and Dal Martello (1977), for example, found that when the pretrained cue was notably less salient than the cue added in the compound training phase, little or no blocking was observed (see also Foree \& LoLordo, 1973). This makes it impossible to make any unequivocal prediction about the nature of any blocking effect observed between landmarks and geometry in the present case. Blocking might be reciprocal, with the landmark blocking geometry and geometry blocking the landmark, in both males and females. But if there were asymmetry in any blocking effect between landmarks and geometry, we would expect to see less blocking of geometry by the landmark than vice versa in males and less blocking of the landmark by geometry than vice versa in females.

In Experiment 2a, two blocking groups (with equal number of males and females in each group) received prior training with a single cue, either the landmark alone in the circular pool or one corner of the triangular pool in the absence of the landmark signaling the location of the platform. In a second phase of the experiment, the two blocking groups and two new control groups (with equal number of males and females in each group) were trained in a compound-cue learning task, with both sources of information simultaneously indicating the platform's position. Final test trials were conducted, without the platform, with only one cue present, either the single landmark in the circular pool or the triangular pool with no landmark present.

\section{Method}

\section{Subjects and apparatus}

The subjects were 64 naive Long Evans rats, 32 males and 32 females, approximately 4 months old at the beginning of the experiment.The animals were kept and maintained as in 
Experiment 1. The apparatus, the experimental room, the platform, the landmark, $\mathrm{X}$, and the two walls forming the triangular-shaped pool were the same as those used in Experiment 1.

\section{Procedure}

There were four types of trials: pretraining, single-cue training, compound-cue training, and tests. The procedure for pretraining was the same as that in Experiment 1 . Training consisted of two phases: single-cue training, or phase 1 , and compound-cue training, or phase 2 . Rats were semirandomly divided into four groups of 16 animals each (i.e., 8 males and 8 females per group): blocking(L), blocking(S), and two control groups that were not trained in phase 1 . The procedure for the training trials of phases 1 and 2 was very similar to that for the pretraining trials, with the exception that one or two cues were always present. The rats were placed in the pool in a different location on each trial, as far as possible equally often with the platform to the right, to the left, or in front of where the rat was placed. These trials had an ITI of $8-10 \mathrm{~min}$, and the platform, landmark, and triangular shape were rotated between trials. In phase 1 , blocking( $(\mathrm{L})$ and blocking( $(\mathrm{S})$ groups received 40 trials of single-cue training for 5 days (8 trials daily). For the blocking(L) group, the landmark, $\mathrm{X}$, was always present in the circular pool, predicting the position of the hidden platform (see Fig. 1a). The blocking(S) group was trained with the two boards forming the triangular shape of the pool, with the point formed by the straight wall to the left and a circular wall to the right predicting the position of the hidden platform (see Fig. 1b). At the end of phase 1, both groups received a test day. This test day consisted of 8 training trials, which were identical to the single-cue training trials for each group, followed by 1 test trial, without the platform, which was $30 \mathrm{~s}$ long. Rats in the blocking(L) group were tested with the landmark $\mathrm{X}$ in the circular pool, while rats in the blocking(S) group were tested with the triangularshaped pool.

In phase 2, all the groups received 24 trials of compoundcue training for 3 days ( 8 trials daily). For all the groups, the position of the platform was now predicted by the two cues, which were presented in compound (as shown in Fig. 1c). At the end of this phase, all the groups received a test day. This test day consisted of 8 training trials, which were identical to the compound-cue training trials, followed by 1 test trial, without the platform, which was $30 \mathrm{~s}$ long. Rats in the blocking(L) group and one control group (control-S) were tested with the triangular-shaped pool, while rats in the blocking(S) group and the second control group (control-L) were tested with the landmark $\mathrm{X}$ in the circular pool. As in Experiment 1 and for purposes of recording the rats' behavior during the tests trials, two areas of the pool were chosen $(0.22 \mathrm{~m}$ in diameter;twice the hidden platform diameter), one in front of the target cue (i.e., either the landmark in the circular pool or the correct corner of the triangular-shaped pool), and the other one, for control reasons, $180^{\circ}$ apart (as shown in Figs. 1d and 1e).

As in the previous experiment, we also examined the possibility that the estrous cycle of female rats could influence their performance (see the Appendix for details).

\section{Results and discussion}

Figure 4 shows the latencies for finding the platform by males and females in all the groups throughout single-cue learning. An ANOVA conducted on the single-cue training phase, with group [blocking(L), blocking(S)], male-female, and days (1-5) as factors, revealed that the days variable was significant, $F(4,112)=64.40$, as well as the group $\times$ days interaction, $F(4,112)=15.57$. Simple main effects of the group $\times$ days interaction showed that the groups differed on day 1 only, $F(1,30)=16.49$, reflecting the fact that the rats trained with the single landmark in the circular pool reached the platform faster than did those trained with the triangular-
Fig. 4 Mean escape latencies by the four groups (i.e., males and females separately) in Experiment 2a during the two phases of training and the test days. Errors bars denote standard errors of the means
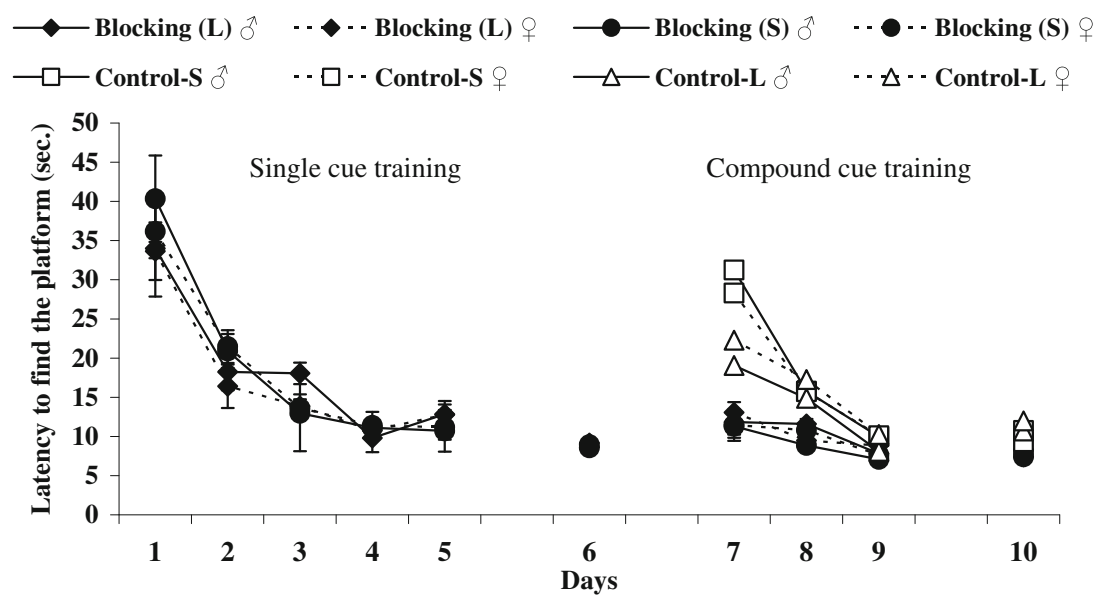
shaped pool. No other main effect or interaction was significant $\left(F_{\mathrm{S}}<0.5\right)$. All the rats improved their performance as training progressed. An ANOVA of the escape trials during the test day (day 6), with group [blocking(L), blocking(S)] and male-female as factors, revealed that no main effect or interaction was significant, $F_{\mathrm{S}}<1$.

On the test trial of phase 1, as in the previous experiment, preliminary Student $t$-tests were used to compare performance of the male and female rats in the blocking groups [i.e., blocking(L) males, blocking(L) females, blocking(S) males, blocking(S) females] in the two areas (target, control) with chance (i.e., 0.58 and $0.88 \mathrm{~s}$ [circular and triangular pools, respectively]) in order to evaluate whether the test results reflected significant spatial learning. All the comparisons differed from chance in the target areas, $t(7)=55.86,38.53$, 27.40, and 44.21 [blocking(L) males, blocking(L) females, blocking(S) males, and blocking(S) females, respectively], while none of them differed from chance in the control areas $(t \mathrm{~s}<0.5)$. Consequently, no further analyses were performed with the results from the control areas. An ANOVA on the time spent in the target areas, with group [blocking(L), blocking(S)] and male-female as factors, revealed that the only significant variable was male-female, $F(1,28)=13.89$, reflecting the fact that males' performance was better than females'. No other main effect or interaction was significant, $F_{\mathrm{s}}<3.0$.

In the compound-cue training phase, the two blocking groups [blocking(L), blocking(S)] continued to perform accurately, while the two control groups (control-S and control-L) rapidly learned to find the platform (see Fig. 4). Although the two control groups obviously reached the platform more slowly than did the two blocking groups, they were faster than the latter groups had been on days $1-3$ in phase $1, F(1,60)=$ 19.25. An ANOVA of the escape trials during the test day (day 10), with group [blocking(L), blocking(S), control-S, and control-L)] and male-female as factors, showed that no main effect or interaction was significant, $F \mathrm{~s}<3.5$.
Figure 5 shows the time spent in the two recording areas (i.e., target and control) by the four main groups (data for the males and females within each group being shown separately) during the 30 -s test trials (i.e., shape test and landmark test [triangular and circular pools, respectively]) of the compound-cue training phase. Preliminary Student $t$ tests were used to compare the performance of each group in the two areas (target, control) with chance (i.e., 0.58 and $0.88 \mathrm{~s}$ [circular and triangular pools, respectively]) in order to evaluate whether the test results reflected significant spatial learning. Males and females in all the groups differed from chance in the target areas, $t(7)=41.30,11.28$, $30.81,13.69,11.33,15.03,20.87$, and 31.09, for blocking(L) males, blocking $(\mathrm{L})$ females, control-S males, control-S females, blocking(S) males, blocking(S) females, control-L males, and control-L females, respectively], while none of them differed from chance in the control areas, $(t<1.5)$. Consequently, no further analyses were performed of the results from the control areas. An ANOVA on the time spent in the target areas, with group (blocking, control), malefemale, and type of cue tested (landmark or shape) as factors, revealed that the group and male-female variables were significant, $F \mathrm{~s}(1,56)=71.21$ and 5.42 , respectively; the male-female $\times$ type of cue tested interaction was also significant, as well as the triple male-female $\times$ cue $\times$ group interaction, $F \mathrm{~s}(1,56)=62.60$ and 19.38 , respectively. The analysis of the triple male-female $\times$ cue $\times$ group interaction showed that on both cues tested (landmark and shape), the group $\times$ male-female interaction was significant, $F \mathrm{~s}(1,28)=$ 6.44 and 14.55 , respectively. Simple main effects of these interactions revealed that on the shape test trial, the group variable was significant in females only, $F(1,14)=23.83$, reflecting the fact that the control group (control-S) spent more time searching in the target area than did the blocking group [blocking(L)]; the two groups did not differ in males, $(F<1)$. On the landmark test trial, the group variable was significant in both sexes, $F \mathrm{~s}(1,14)=43.94$ and 10.49 ,
Fig. 5 Mean time spent in the two recording areas (i.e., target and control) by the four groups (i.e., males and females separately) during the test trials (i.e., shape test and landmark test [triangular and circular pools, respectively]) of the compound cue training phase in Experiment 2a. Errors bars denote standard errors of the means

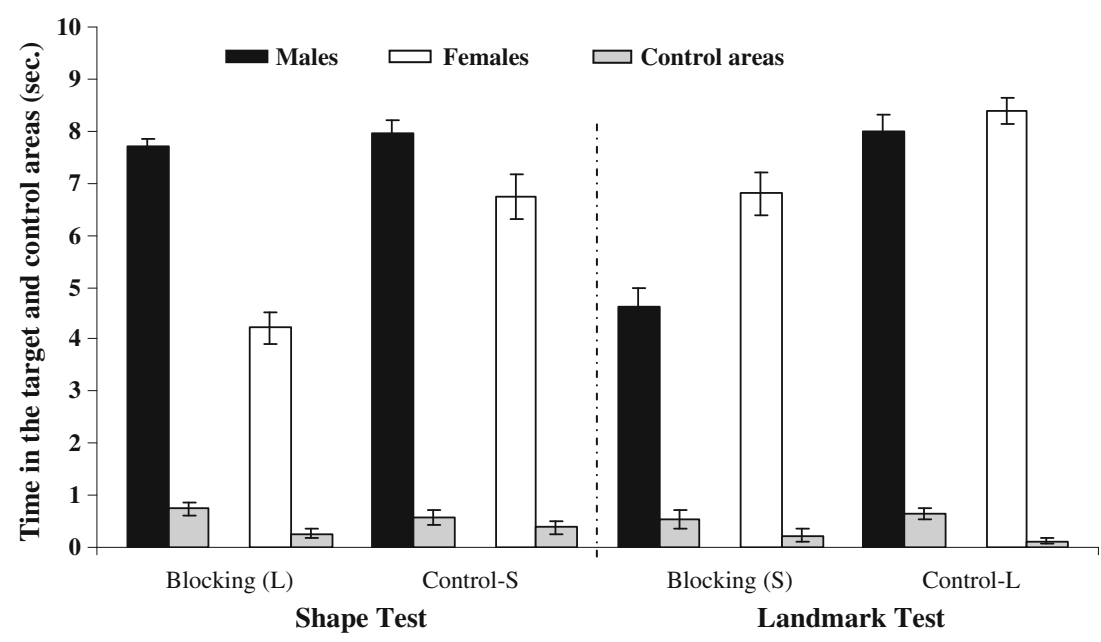


respectively, reflecting the fact that in both sexes, the control group (control-L) spent more time searching in the target area than did the blocking group [blocking(S)]. These results indicate that landmark learning blocked shape learning in females, but not in males, while shape learning blocked landmark learning both in males and in females. Moreover, looking at Fig. 5, there is an apparent suggestion that the magnitude of the blocking effect in females was greater in the group tested on the shape of the pool than in the group tested on the landmark; this would complement the results with males, where the blocking effect was clearly greater in animals tested on the landmark than in animals tested on shape of the pool. However, the interaction in females was not significant $(p=.18)$.

In summary, both males and females showed significant blocking, but whereas in females the blocking effect was reciprocal, with geometry blocking landmark learning and the landmark blocking learning about geometry, in males blocking was asymmetrical: Prior training on geometry blocked learning about the landmark, but prior training on the landmark failed to block learning about geometry.

\section{Experiment 2b}

The results of Experiment 2a revealed a reciprocal blocking effect between the landmark and geometry in females only. In males, prior training with geometry blocked landmark learning, but prior training with the landmark did not block learning about the geometric cue. In Experiment 2b, we attempted to increase the chance that landmark learning could block shape learning in males by giving extended initial training with the landmark. In a related blocking experiment, Horne and Pearce (2009) suggested that male rats might need more experience with a landmark (in their study, a sphere located immediately above a hidden platform) before it could block learning about geometry. They found that 96 trials of initial training, with the landmark alone signaling the location of the platform, blocked subsequent learning about a geometrical cue when the location of the platform was also signaled by one corner of a triangular-shaped pool. No blocking was observed, however, after only 48 trials of initial training with the landmark. Consequently, the aim of Experiment $2 b$ was to evaluate whether an extended training with the landmark (i.e., 80 trials instead of 40 trials, as in Experiment 2a) could affect subsequent learning about the shape of the pool when both sources of information simultaneously indicated the platform's position. The experiment was conducted with two groups of male rats only, a blocking and a control group.

Method

\section{Subjects and apparatus}

The subjects were 16 naive Long Evans male rats, approximately 3 months old at the beginning of the experiment. The animals were kept and maintained as in Experiment 1 and 2a. The apparatus, the experimental room, the platform, the landmark, $X$, and the two walls forming the triangular-shaped pool were the same as those used in Experiment 1 and 2a.

\section{Procedure}

The general procedure was the same as that in Experiment 2a, with the main exception that, in this experiment, the escape trials of the single-cue training phase were doubled (i.e., 80 escape trials were conducted, instead of the 40 escape trials in Experiment 1a). Rats were semirandomly divided into two groups (i.e., 8 rats per group), which were the same as those in Experiment 2a: blocking(L) and control-S.

\section{Results and discussion}

Figure 6 shows the latencies for finding the platform by the two groups throughout cue learning. An ANOVA conducted on the single-cue training phase revealed that the days variable was significant, $F(9,63)=26.87$. All the rats improved their performance as training progressed.
Fig. 6 Mean escape latencies for the two groups in Experiment $2 \mathrm{~b}$ during the two phases of training and the test days. Errors bars denote standard errors of the means

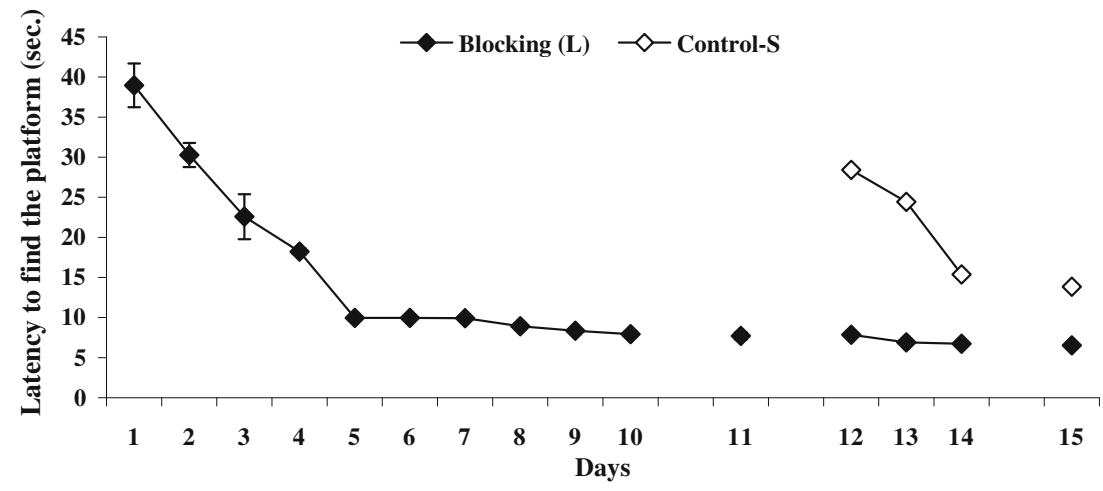


On the test trial of phase 1 , a $t$-test was used to compare pretrained rats' performance in the target area with that in the control area. The time spent in the target area differed significantly from that spent in the control area, $t(7)=$ 36.69 , which gave evidence of significant spatial learning. In the compound-cue training phase, the blocking group [blocking(L)] continued to perform accurately, while the new group (control-S) rapidly learned to find the platform (see Fig. 6). Although rats in the control group obviously reached the platform more slowly than did rats in the blocking group, they were faster than the latter rats had been on days $1-3$ in phase $1, F(1,14)=6.67$. An ANOVA of the escape trials during the test day (day 15) showed that the groups differed, $F(1,14)=269.42$, reflecting the fact that rats in the blocking group reached the platform faster than did those in the control group.

Figure 7 shows the time spent in the two recording areas (i.e., target and control) by the two groups during the 30 -s test trial (i.e., shape test in the triangular pool) in the compoundcue training phase. Preliminary Student $t$-tests were used to compare the performance of the two groups in the two recording areas (target, control) with chance (i.e., $0.58 \mathrm{~s}$ ) in order to evaluate whether the test results reflected significant spatial learning. Both groups differed from chance in the target area, $t(7)=14.38$, and 12.42 (blocking and control groups, respectively), while none of the groups differed from chance in the control area, $(t<-2)$. Consequently, no further analyses were performed of the results for the control area. An ANOVA on the time spent in the target area revealed that the groups differed, $F(1,14)=14.12$, reflecting the fact that the controlgroup spent more time searching in the target area than did the blocking group. This result clearly indicates that landmark learning blocked shape learning.

In summary, the results of this experiment revealed that after an extended single-cue training with the landmark, landmark learning could block shape learning in male rats.

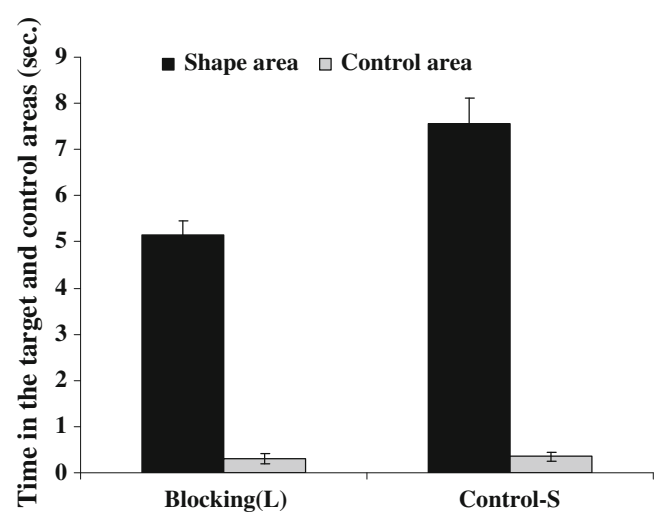

Fig. 7 Mean time spent in the two recording areas (i.e., target and control) by the two groups during the test trial (i.e., shape test in the triangular pool) of the compound-cue training phase of Experiment $2 \mathrm{~b}$. Error bars denote standard errors of the means

\section{General discussion}

In Experiment 1, rats were trained with the platform next to a distinctive landmark in a circular pool, with the platform in one corner of a triangular pool with no landmark, or with both sources of information signaling the location of the platform. The hypothesis tested was whether the relative salience of these two cues for males and females would affect the direction of overshadowing. The results showed an asymmetrical overshadowing effect in both sexes. In males, geometry overshadowed landmark learning, but landmark learning did not overshadow learning about geometry, while in females, landmark learning overshadowed learning about geometry, but geometry learning did not overshadow landmark learning. It was the more discriminable, salient, or preferred source of information (geometry for males and landmark for females, as shown by Rodríguez et al., 2010) that overshadowed the less discriminable, salient, or preferred cue.

We should acknowledge the possibility that these overshadowing results are due to generalization decrement (see Pearce, 1994; Wagner, 2003). Put simply, the argument is that animals trained with a compound cue may perform less accurately when tested with either component alone than animals trained with these components in isolation, because responding established to one cue (the compound) does not transfer perfectly to a different cue (either component). Overshadowing is asymmetrical because the removal of the more salient cue for the test produces a greater perceptual change than does removal of the less salient cue. We do not have any control for this possibility. But that hardly detracts from the main point of the experiment, which was to test whether the difference between males and females in the relative salience of geometrical and landmark cues would affect the direction of asymmetrical overshadowing. It clearly did. Moreover, generalization decrement will not explain the occurrence of blocking.

Experiment 2a demonstrated reciprocal blocking of geometry by a landmark and of the landmark by prior training on geometry, but only in females. In males, prior training on geometry blocked learning about the landmark, but not vice versa. It was only after extended prior training with the landmark alone in Experiment $2 b$ that it was able to block learning about geometry. These findings with male rats nicely confirm those reported by Horne and Pearce (2009).

Taken together, our results clearly support the contention that, as others have observed (e.g., Graham, Good, McGregor, \& Pearce, 2006; Horne \& Pearce, 2009; Pearce, Graham, Good, Jones, \& McGregor, 2006), cue competition between geometry and other visual cues does sometimes occur when rats are trained to navigate toward a hidden platform in the Morris pool. As we noted in the introduction, however, there 
have been rather more numerous failures to find any evidence of blocking or overshadowing in such experiments. Do our data shed any light on these discrepancies? In principle, they surely make it less surprising that experiments with male rats should often show no evidence of overshadowing (or even of blocking) of geometrical cues by landmarks (e.g., Hayward, Good, \& Pearce, 2004; Hayward, McGregor, Good, \& Pearce, 2003; McGregor et al., 2009; Pearce, Ward-Robinson, Good, Fussell, \& Aydin, 2001; Wall, Botly, Black, \& Shettleworth, 2004) and suggest that different results might have been observed with female rats.

It would be foolish, however, to pretend that our data have resolved all problems in the existing literature. Unsurprisingly, others have considered the possibility that the failure of a landmark to overshadow or block learning about geometry occurred because the landmark was so much less salient than the geometrical cue (Hayward et al., 2004; Hayward et al., 2003). However, Hayward et al., who assessed salience on the basis of the performance of groups trained with each cue in isolation, found no evidence to support this. It remains to be seen whether the measure of salience employed by Rodríguez et al. (2010) - namely, opposing the two cues and seeing which one wins-would have given the same result. An even more puzzling finding, from our point of view, is that McGregor et al. (2009) found not only that landmarks did not overshadow geometry, but also that geometry failed to overshadow landmarks. It is obvious that the experiments reported here do not resolve all discrepancies in the literature; it could be argued, indeed, that they have added further discrepancies. At the same time, however, they do suggest that the frequently reported failure of landmarks to overshadow or block learning about geometry in male rats may be less surprising than has sometimes been thought.

Acknowledgements This research was supported by a grant from the Spanish 'Ministerio de Educación y Ciencia' "(Ref. PSI201020424) to V.D.Chamizo. The authors are very grateful to Ariadna Arbat and Mónica Plata for excellent help in running the subjects.

\section{Appendix: Determination of the estrous cycle}

\section{Synchronization of groups}

The rats were examined to establish the estrous cycle by a daily collection of a vaginal smear for 8 days before the start of Experiment 1 and 2a. In order to establish two distinct synchronized groups of females ( $n=4$ per group always) according to the different phases of the estrous cycle, the Whitten effect (Whitten, 1966) was carried out, which produces the synchronization of the estrous cycle in females by the exposure to male pheromones. Specifically,

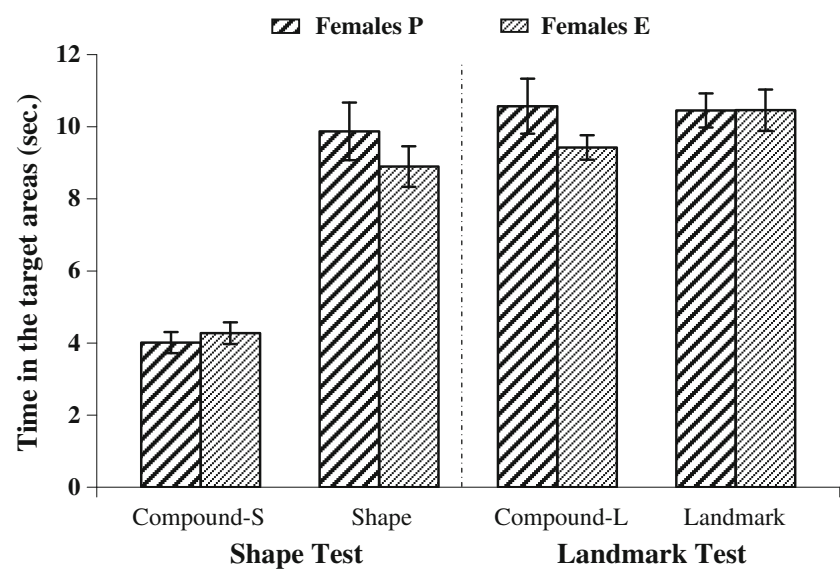

Fig. 8 Mean time spent in the target areas by the female subjects (proestrus and estrus) in the four groups (compound-S, compound-L, shape, and landmark) during the test trials (i.e., shape test and landmark test [triangular and circular pools, respectively]) in Experiment 1. Error bars denote standard errors of the means

some shavings soaked in urine and feces of male rats were introduced in half of the cages of the female rats before the pretraining phase. During the experiment, they continued to be examined every day. On the single test day (Experiment 1) or on the last test day (Experiment 2a), rats were examined both pre- and post-testing to ensure that they did not change over to the next estrous cycle phase during testing.

\section{Vaginal examination}

We performed vaginal examination following the procedure used by Marcondes, Bianchi, and Tanno (2002): The females had their tails gently raised, and then a cotton swab, previously soaked in saline, was inserted into their vaginas to obtain the cytology by circular movements. The

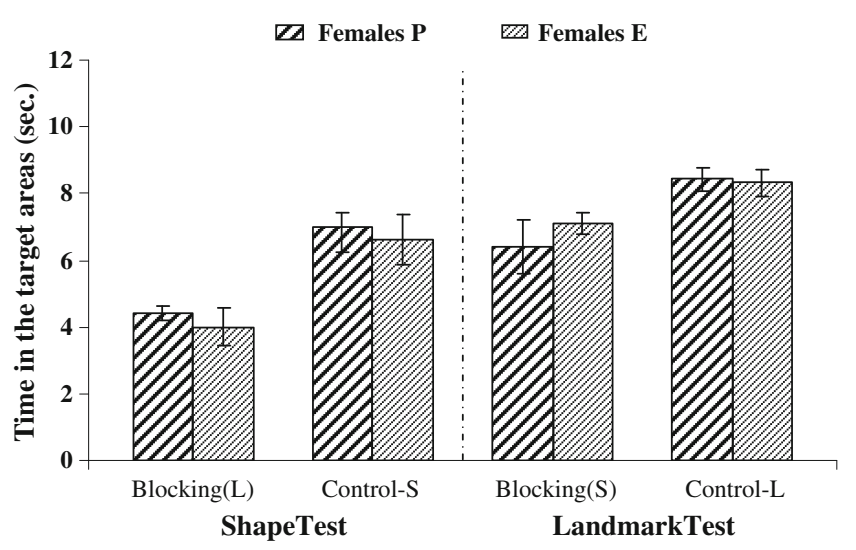

Fig. 9 Mean time spent in the target areas by the female subjects (proestrus and estrus) in the four groups [blocking(L), blocking(S), control-S, and control-L] during the test trials (i.e., shape test and landmark test [triangular and circular pools, respectively]) of the compound-cue training phase in Experiment 2a. Error bars denote standard errors of the means 
product of this cytology was examined under a light microscope $(10 \times$ objective) to determine the phase of the estrous cycle in which each animal was, following the procedures used in Sava and Markus (2005) and Feder (1981). The proestrus was defined as a predominance of epithelial or nucleated cells, the estrus as a predominance of cells without nuclei, or cells cornified, the metaestrus as a combination of cornified cells and leukocytes, and diestrus as a predominance of leukocytes. The female rats were divided into two subgroups: proestrus if $50 \%-70 \%$ of visible cells were nucleated (i.e., epithelial) and estrus if nucleated cells were less than $5 \%$. Furthermore, to minimize the effects that the manipulation described above might result in females, male rats received a similar handling: They were turned upside down to expose the perineal region, and then the scrotum was wiped with a cotton swab.

\section{Experiment 1}

In order to see whether there was any effect of the estrous cycle on females' test performance, a further ANOVA was conducted on the females' test scores only, with the additional factor of proestrus/estrus (see Fig. 8). There was no main effect of estrous cycle, nor any interaction with any other factor, $(F<2.5)$. The implication is that the previous overshadowing results do not seem to be influenced by the females' estrous cycle.

\section{Experiment 2a}

In order to see whether there was any effect of the estrous cycle on females' test performance, a further ANOVA was conducted on the females' test scores only (i.e., on the last test day), with the additional factor of proestrus/estrus (see Fig. 9). There was no main effect of estrous cycle, nor any interaction with any other factor, $(F<2)$. The implication is that the previous blocking results do not seem to be influenced by the females' estrous cycle.

\section{References}

Chai, X. J., \& Jacobs, L. F. (2009). Sex differences in directional cue use in a virtual landscape. Behavioral Neuroscience, 123, 276283.

Cheng, K. (1986). A purely geometric module in the rat's spatial representation. Cognition, 23, 149-178.

Feder, H. H. (1981). Estrous cyclicity in mammals. In N. J. Adler (Ed.), Neuroendocrinology of reproduction physiology and behavior (pp. 279-348). New York: Plenum.

Foree, D. D., \& LoLordo, V. M. (1973). Attention in the pigeon: Differential effects of food-getting versus shock-avoidance procedures. Journal of Comparative and Physiological Psychology, 85, 551-558.
Gallistel, C. R. (1990). The organization of learning. Cambridge, MA: MIT Press.

Graham, M., Good, M. A., McGregor, A., \& Pearce, J. (2006). Spatial learning based on the shape of the environment is influence by properties of the objects forming the shape. Journal of Experimental Psychology:Animal Behavior Processes, 32, 44-59.

Hall, G., Mackintosh, N. J., Goodall, G., \& DalMartello, M. (1977). Loss of control by a less valid or by a less salient stimulus compounded with a better predictor of reinforcement. Learning and Motivation, 8, 145-158.

Hayward, A., Good, M. A., \& Pearce, J. M. (2004). Failure of a landmark to restrict spatial learning based on the shape of the environment. Quarterly Journal of Experimental Psychology, $57 B, 289-314$.

Hayward, A., McGregor, A., Good, M. A., \& Pearce, J. M. (2003). Absence of overshadowing and blocking between landmarks and the geometric cues provided by the shape of a test arena. Quarterly Journalof Experimental Psychology, 56B, 114-126.

Horne, M. R., \& Pearce, J. M. (2009). A landmark blocks searching for a hidden platform in an environment with a distinctive shape after extended pretraining. Learning \& Behavior, 37, 167-178.

Kamin, L. J. (1969). Predictability, surprise, attention and conditioning. InB.A. Campbell \&R. M. Church (Eds.), Punishment and aversive behavior(pp. 279-296). New York: Appleton-CenturyCrofts.

Mackintosh, N. J. (1976). Overshadowing and stimulus intensity. Animal Learning and Behavior, 4, 186-192.

Marcondes, F. K., Bianchi, F. J., \& Tanno, A. P. (2002). Determination of the estrous cycle phases of rats: Some helpful considerations. Brazilian Journal of Biology, 62, 609-614.

McGregor, A., Horne, M., Esber, G. O., \& Pearce, J. M. (2009). Absence of overshadowing between a landmark and geometric cues in a distinctively shaped environment: A test of Miller and Shettleworth (2007). Journal of Experimental Psychology: Animal Behavior Processes, 35, 357-371.

Morris, R. G. M. (1981). Spatial localization does not require the presence of local cues. Learning and Motivation, 12, 239-260.

Pavlov, I. P. (1927). Conditioned reflexes. Oxford: Oxford University Press.

Pearce, J. M. (1994). Similarity and discrimination: A selective reviewand a connectionist model. Psychological Review, 101, 587-607.

Pearce, J. M. (2009). An associative analysis of spatial learning. Quarterly Journal of Experimental Psychology, 62, 1665-1684.

Pearce, J. M., Graham, M., Good, M. A., Jones, P. M., \& McGregor, A. (2006). Potentiation, overshadowing, and blocking of spatial learning based on the shape of the environment. Journal of Experimental Psychology:Animal Behavior Processes, 32, 201-214.

Pearce, J. M., Ward-Robinson, J., Good, M., Fussell, C., \& Aydin, A. (2001). Influence of a beacon on spatial learning based on the shape of the test environment. Journal of Experimental Psychology: Animal Behavior Processes, 27, 329-344.

Rodríguez, C. A., Torres, A. A., Mackintosh, N. J., \& Chamizo, V. D. (2010). Sex differences in preferential strategies to solve a navigation task. Journal of Experimental Psychology: Animal Behavior Processes, 36, 395-401.

Roof, R. L., \& Stein, D. G. (1999). Gender differences in Morris water maze performance depend on task parameters. Physiology and Behavior, 68, 81-86.

Saucier, D. M., Green, S. M., Leason, J., MacFadden, A., Bell, S., \& Elias, L. J. (2002). Are sex differences in navigation caused by sexually dimorphic strategies or by differences in the ability to use the strategies? Behavioral Neuroscience, 116, 403-410.

Sava, S., \& Markus, E. J. (2005). Intramaze cue utilization in the water maze: Effects of sex and estrous cycle in rats. Hormones and Behavior, 48, 23-33. 
Wagner, A. (2003). Context-sensitive elemental theory. Quaterly Journal of Experimental Psychology, 56B, 7-29.

Wall, P. L., Botly, L. C. P., Black, C. K., \& Shettleworth, S. J. (2004).

The geometric module in the rat: Independence of shape and feature learning in a food finding task. Learning and Behavior, 32, 289-298.
Whitten, W. K. (1966). Pheromones and mammalian reproduction. Advances in Reproductive Physiology, 1, 155-177.

Williams, C. L., Barnett, A. M., \& Meck, W. H. (1990). Organizational effects of early gonadal secretions on sexual differentiation in spatial memory. Behavioral Neuroscience, 104, 84-97. 